\title{
ON UNIVERSAL MAPPINGS AND FREE TOPOLOGICAL GROUPS
}

\author{
P. SAMUEL
}

It has been observed ${ }^{1}$ that constructions so apparently different as Kronecker products, extension of the ring of operators of a module, field of quotients of an integral domain, free groups, free topological groups, completion of a uniform space, Cech compactification enter in the same frame. We intend in this paper to explain a rather general process of construction which may be applied to most of the examples quoted above.

This paper will proceed axiomatically. In fact the problem under question (problem of a "universal mapping") can be only stated after a certain number of axioms. When the method of construction has been explained we shall illustrate it by the classical example of the completion of a uniform space. For more examples the reader is referred to a forthcoming book of N. Bourbaki. The same method gives also necessary and sufficient conditions for many imbedding problems. Both topological and algebraic examples will be given. In the last part of the paper our method of construction will be applied to Markoff's theory of free topological groups. ${ }^{2}$

1. Problems of universal mappings. Given a set $E$ it is possible to define on it certain kinds of structures, that is structure of ring, field, topological space. ${ }^{3}$ We shall denote by $S$ or $T$ certain kinds of structures. A set with a structure $T$ will be called a $T$-set: if $T$ is the structure of group the $T$-sets are the groups. An isomorphism for the structure $T$ will be called a $T$-isomorphism:

$T$-mappings. Induced structures. Given a kind of structure $T$ it happens very often that, for every pair $E_{1} E_{2}$ of $T$-sets, there has been defined a family of mappings of $E_{1}$ into $E_{2}$ satisfying the following axioms:

$\mathrm{A}_{1}$. Every $T$-isomorphism is a T-mapping.

$\mathrm{A}_{2}$. If $f: E_{1} \rightarrow E_{2}$ and $g: E_{2} \rightarrow E_{3}$ are $T$-mappings, then the composite

Received by the editors August 12, 1947.

${ }^{1}$ Unpublished manuscripts of N. Bourbaki.

2 Markoff, Bull. Acad. Sci. USSR. vol. 9 (1945) pp. 3-64.

${ }^{3}$ For precise definitions of the words "structure," "kind of structure," "isomorphism" see N. Bourbaki, Theorie des ensembles (Résultats), Part 10, Paris, Herrmann, 1939. 
mapping $g \circ f: E_{1} \rightarrow E_{3}$ is a $T$-mapping.

$\mathrm{A}_{3}$. A necessary and sufficient condition for a one-to-one mapping $f$ of $E_{1}$ onto $E_{2}$ to be a $T$-isomorphism is that $f$ and $f^{-1}$ be $T$-mappings.

EXAMPLE. If $T$ is the structure of group the $T$-mappings are the homomorphisms; if $T$ is the structure of topological space the $T$-mappings are the continuous ones.

Let now $\sigma$ and $\sigma^{\prime}$ be two structures $T$ defined on $E$ and $E^{\prime} \subset E$ respectively. We shall say that $\sigma^{\prime}$ is induced by $\sigma$ when:

$\mathrm{I}_{1}$. The injection of $E^{\prime}$ into $E$ is a $T$-mapping.

I. $_{2}$ If $f: F \rightarrow E$ is a $T$-mapping and if $f(F) \subset E^{\prime}$, then $f$ considered as mapping of $F$ into $E^{\prime}$ is a $T$-mapping.

$I_{1}, I_{2}$ and $A_{3}$ show immediately the uniqueness of the induced structure.

If $E^{\prime} \subset E$ is capable of an induced structure we shall say that $E^{\prime}$ is $T$-closed. We suppose that the following axioms hold:

$\mathrm{S}_{1}$. A subset of $E$ composed of all the elements where a family of $T$-mappings takes the same value is T-closed.

$\mathrm{S}_{2}$. Any intersection of $T$-closed sets is $T$-closed.

$S_{2}$ is a consequence of $S_{1}$ if we allow mappings which are not everywhere defined: The notion of $T$-closed set is obviously transitive. $S_{2}$ allows us to define the $T$-closure $\bar{E}^{\prime}$ of a subset $E^{\prime} \subset E$ as the intersection of all $T$-closed subsets containing $E^{\prime}$. We shall suppose:

$\mathrm{S}_{3}$. Cardinal $\left(\bar{E}^{\prime}\right) \leqq$ certain function of cardinal $\left(E^{\prime}\right)$, a function which depends only on the structure $T$.

In most cases the function " $2^{2^{\text {card }}\left(E^{\prime}\right)}$ " will be sufficient.

Axioms for the cartesian products. In many important cases it is possible, given a family $\left(E_{\alpha}\right)$ of $T$-sets, to define on the cartesian product $\prod_{\alpha} E_{\alpha}$ a structure $T$ which satisfies the following conditions:

$\mathrm{P}_{1}$. The projections (on the components) are T-mappings.

$\mathrm{P}_{2}$. If the $f_{\alpha}: E \rightarrow E_{\alpha}$ are $T$-mappings, the product mapping $f: E$ $\rightarrow \prod_{\alpha} E_{\alpha}$ (defined by $f(x)=\left(f_{\alpha}(x)\right)$ ) is a T-mapping.

As easy consequences: the projections on the partial products are $T$-mappings; these partial products are $T$-closed; the given structure of the "coordinate axis" is induced by the product structure. Applying $\mathrm{A}_{3}$ we see also that the product structure is unique.

Remark. The example of the structure of topological space shows easily that the axioms $A, S, P$ are independent.

Statement and solution of the problem of universal mappings. Given two kinds of structures $S$ and $T$, suppose we have defined the $T$-mappings, and also mappings of $S$-sets into $T$-sets, called the $(S-T$ mappings, denoted by greek letters, and satisfying:

$(\mathrm{S}-\mathrm{T})_{1}$. The composite mapping $f \circ \phi$ of an $(S-T)$-mapping $\phi$ and of 
a T-mapping $f$ is an ( $S$-T)-mapping.

$(\mathrm{S}-\mathrm{T})_{2}$. The product mapping of a family of (S-T)-mappings is an $(S-T)$-mapping.

The structure $T$ is supposed to satisfy the axioms $\mathrm{A}, \mathrm{S}$ and $\mathrm{P}$.

The problem we have in view ("problem of universal mappings" or "problem U") is the following: given any $S$-set $E$ to find a $T$-set $F_{0}$ and an $(S-T)$-mapping $\phi_{0}$ of $E$ into $F$ such that:

$\left(\mathrm{U}_{1}\right)$. Every $(S-T)$-mapping $\phi$ of $E$ into any $T$-set $F$ has the form $\phi=f \circ \phi_{0}$ where $f$ is a $T$-mapping of $F_{0}$ into $F$.

It is clear that, if such an $F_{0}$ exists, the $T$-closure of $\phi_{0}(E)$ in $F_{0}$ will also satisfy $\left(U_{1}\right)$. Therefore $\left(S_{1}\right)$ there will exist a pair $\left(F_{0}, \phi_{0}\right)$ such that:

$\left(\mathrm{U}_{2}\right)$. Two $T$-mappings of $F_{0}$ into $F$ which coincide on $\phi_{0}(E)$ are identical.

We deduce immediately from $A_{3}$ that a pair $\left(F_{0}, \phi_{0}\right)$ satisfying $\left(U_{1}\right)$ and $\left(\mathrm{U}_{2}\right)$ is uniquely determined up to isomorphisms.

We now come to the construction of a pair $\left(F_{0}, \phi_{0}\right)$ satisfying $\left(U_{1}\right)$. Consider the set of all $(S-T)$-mappings of $E$ into all $T$-sets whose cardinal does not exceed the one indicated in $S_{3}\left(2^{2^{\text {oard }} E}\right.$ in most cases). Let $\left\{\phi_{\alpha}\right\}$ be this set, $\phi_{\alpha}$ mapping $E$ into $F_{\alpha}$. Let $F_{0}=\prod_{\alpha} F_{\alpha}$, and $\phi_{0}$ be the product mapping $x \rightarrow\left(\phi_{\alpha}(x)\right)$ of $E$ into $F_{0} . F_{0}$ is a $T$-set $(P)$, and $\phi_{0}$ an (S-T)-mapping $\left((S-T)_{2}\right)$. Let $\phi$ be an $(S-T$-) mapping of $E$ into $F, F^{\prime}$ the $T$-closure of $\phi(E)$ in $F, i$ the injection of $F^{\prime}$ in $F$. By $\mathrm{S}_{3}, \mathrm{I}_{2}$ and (S-T) the contraction $\phi^{\prime}$ of $\phi$, mapping of $E$ into $F$, is among the $\phi_{\alpha}$, say $\phi_{\alpha_{0}}$. Let $p_{0}$ be the projection of $\prod_{\alpha} F_{\alpha}$ onto $F_{\alpha_{0}}$. We may write $\phi=i \circ \phi^{\prime}=i \circ \phi_{\alpha_{0}}=i \circ p_{0} \circ \phi_{0}$. Since $i \circ p_{0}$ is a $T$-mapping of $\prod_{\alpha} F_{\alpha}$ into $F$, the pair $\left(F_{0}, \phi_{0}\right)$ satisfies $\left(\mathrm{U}_{1}\right)$. Q.E.D.

EXAMPLE. The preceding construction applies to all the examples quoted in the introduction except the field of quotients of an integral domain (a product of fields being not a field). For these examples the reader is referred to a forthcoming book of $\mathrm{N}$. Bourbaki.

We shall give only the example of the completion of a uniform space. ${ }^{4} S$ is the structure of separated uniform space, $T$ the structure of complete separated uniform space. The $T$ - and $(S-T)$-mappings are the uniformly continuous ones. All our axioms are verified. Therefore, given a separated uniform space $E$, the preceding construction provides with a complete space $F_{0}$ and a uniformly continuous mapping $\phi_{0}$ of $E$ into $F$ such that every uniformly continuous mapping of $E$ is "induced" by a uniformly continuous mapping of $F_{0}$.

We shall prove that, in this case, $\phi_{0}$ is a uniform structural isomorphism of $E$ onto $\phi_{0}(E)$. In fact consider the family $E$ of all uni-

${ }^{4}$ N. Bourbaki, Topologie générale, chap. 2, Paris, Hermann, 1940. 
formly continuous distances (not necessarily satisfying the separation axiom " $d(x, y)=0$ implies $x=y$ ") on $E$. Since the real line is a complete space the function $f$ defined by fixing one of the arguments in the distance $d$ is among the $f_{\alpha}$. Since the uniform structure of $E$ may be defined by the family $(\mathbb{E}, 5$ we see immediately-denoting by $\left\{f_{\beta}\right\}$ the subset of $\left\{f_{\alpha}\right\}$ composed of all mappings deduced from distances, and by $\pi$ the projection of $\prod_{\alpha} F_{\alpha}$ onto $\prod_{\beta} F_{\beta}$ that $\pi \circ \phi_{0}$ is an isomorphism. Hence $\phi_{0}$ is one-to-one and $\phi_{0}^{-1}=\left(\pi \circ \phi_{0}\right)^{-1} \circ \pi$ is uniformly continuous. Hence $\phi_{0}$ is an isomorphism.

By definition of the $T$-closure (smallest complete, that is, closed subspace), we see that $\phi_{0}(E)$ is dense in $F$. We have therefore proved the existence and the uniqueness of the completion of a uniform space (provided we have defined the real line without completion, for example by the cuts process). If $E$ is not separated one verifies easily that $\phi_{0}(E)$ is the associated separated space.

2. Imbedding problems. It of ten happens that the structure $T$ is "richer" than the structure $S$, that is, that there exists a canonical process for giving a structure $S$ to a $T$-set. In an example where $\phi_{0}(E)$ is, in the $S$-set $F_{0}$, capable of an induced structure $S$, arises, with the problem (U), an "imbedding problem": may we consider $E$ as a subset of a set $F$, subset whose structure $S$ is induced by the structure $S$ of $F$ canonically deduced from a structure $T$ ? We shall suppose that a $T$-mapping is also an $S$-mapping for the deduced structures $S$. As a consequence $\left(\mathrm{A}_{3}\right.$ for $\left.S\right)$ the operations "induced structure" and "deduced structure" commute.

If $\phi_{0}(E)$ is capable of an induced structure $S$, and if $\phi_{0}$ is an $S$-isomorphism, the imbedding problem is solved by $\left(F_{0}, \phi_{0}\right)$. Let conversely $(F, \phi)$ be a solution of the imbedding problem, $\phi$ being an $(S-T)$-mapping. We can write $\phi=f \circ \phi_{0}, f$ being a $T$-mapping of $F_{0}$ into $F$. Since $\phi$ is an $S$-isomorphism, and since $f \mid \phi_{0}(E)$ and $\phi_{0}$ are $S$-mappings, it follows from $\mathrm{A}_{2}$ and $\mathrm{A}_{3}$ (for $S$ ) that $f \mid \phi_{0}(E)$ and $\phi_{0}$ are $S$-isomorphisms. Therefore $\left(F_{0}, \phi_{0}\right)$ gives also a solution of the imbedding problem.

We may therefore consider $F$ as a set of equivalence classes in $F_{0}$, the sets $\{a\}\left(a \in \phi_{0}(E)\right)$ being equivalence classes. The fact that this identification is not always trivial (and that the imbedding problem does not admit a unique solution) is shown by the example of the compactifications of a uniform space ( $F_{0}$ being the Cech compactification). Therefore: $A$ necessary and sufficient condition for the imbedding problem of $E$ to be possible is that $\phi_{0}$ be an S-isomorphism.

${ }^{5}$ N. Bourbaki, Topologie générale, chap. 9, part 1, Theorem 1, 1948. 
ExAmples. (1) Characterization of uniformizable spaces. ${ }^{6}$ The structures $S$ and $T$ are the structures of topological space and of compact space respectively. The $S-, T-,(S-T)$-mappings are the continuous ones. Let $\mathfrak{I}$ be the given topology on $E, \mathfrak{I}^{\prime}$ the one induced on $\phi_{0}(E)$ by the product space topology. $\mathfrak{I}^{\prime}$ and $\mathfrak{T}_{0}=\phi_{0}^{-1}\left(\mathfrak{T}^{\prime}\right)$ are uniformizable. In general $\mathfrak{I}$ is finer than $\mathfrak{I}_{0}$. A necessary and sufficient condition for $\mathfrak{I}$ to be uniformizable is that $\mathfrak{T}=\mathfrak{I}_{0}$. Then $F_{0}$ is the Cech compactification of $E$. Using the fact that every compact space may be imbedded in some, finite or transfinite, cube, one shows easily that the condition " $\mathfrak{T}=\mathfrak{T}_{0}$ " is equivalent with the complete regularity of $E$.

(2) Alexandroff's $T_{2}$-space. ${ }^{7} S$ is the structure of topological space, $T$ the structure of $T_{2}$-space, the $S$-, $T$-, $(S-T)$-mappings are the continuous ones. Then $\phi_{0}(E)$ is the "greatest possible" identification space of $E$ which is a $T_{2}$-space.

Remark. It may happen, in some cases where our axioms are not all fulfilled (in particular $\mathrm{S}_{2}$ relative to intersections of $T$-closed sets), that one can however construct $\phi_{0}$, while $F_{0}$ is "too big."

(1). Let $S$ be the structure of ring, $T$ the structure of semi-simple ring. The $S$-, $T$-, $(S-T)$-mappings are the homomorphisms into. In this case $\phi_{0}^{-1}(\{0\})$ is the extension radical $\Re$ of the ring $E .^{8}$ If $\Re=0, E$ may be imbedded in a semi-simple ring and conversely. The structure of $\Re$ may be obtained by studying a sufficient number of homomorphisms of $E$ into semi-simple rings. Let $\mathfrak{a}$ be the twosided ideal $T \cap \bigcap_{p} p E$ ( $T$ : ideal of the elements of finite order in the additive group of $E$; $p$ : prime number). Every semi-simple ring being contained in a product of full matrix rings over $s$-fields, it is clear that $\phi(\mathfrak{a})=0$ for every homomorphism $\phi$ of $E$ into a semisimple ring. Hence $a \subset \Re$. On the other hand the rings $E / p E$ and the Kronecker product $E / T \otimes Q$ of $E / T$ by the rational field $Q$ are algebras over the prime fields. But every algebra is a subalgebra of a full matrix algebra (adjoin a unit element, and consider the left regular representation). Hence $\Re \subset p E, \Re \subset T$. Therefore $\Re=\mathfrak{a}$.

(2) If we restrict the $(S-T)$-mappings to be the homomorphisms onto semi simple rings, it is easily seen that $\phi_{0}^{-1}(\{0\})$ is the radical of $E .^{9}$

${ }^{6} \mathrm{P}$. Samuel, Ultrafilters and compactification of uniform spaces, Princeton thesis, 1947.

${ }^{7}$ P. Alexandroff, Bikompakte Erweiterungen von Räumen, Rec. Math. (Mat. Sbornik) N.S. (1939).

8 O. Goldman. Semi-simple extensions of rings, Bull. Amer. Math. Soc. vol. 52 (1946) pp. 1028-1032.

$9 \mathrm{~N}$. Jacobson, Radical and semi simplicity for arbitrary rings, Amer. J. Math. vol. 67 (1945) pp. 300-320. 
3. Free topological groups. As a more elaborate example of our general method of construction, we shall give a sketchy treatment of Markoff's theory of free topological groups. Only very easy proofs have been omitted.

$S$ is the structure of topological space, $T$ the structure of separated topological group (abelian, or precompact, if desired). The $(S-T)$ mappings are the continuous ones, the $T$-mappings are the continuous homomorphisms. All the axioms A, S, P are satisfied. Applying the general construction to the topological space $E$, we get for $F_{0}$ a separated topological group (abelian, or precompact, if desired) denoted $G(E),(G A(E), G C(E))$. By construction $G(E)$ is such that:

(1) For every continuous mapping $\phi$ of $E$ into a topological group $G$, there exists a continuous homomorphism $\mathrm{g}$ of $G(E)$ into $G$ such that $\phi=g \circ \phi_{0}$.

(2) Two continuous homomorphisms of $G(E)$ into a topological group $H$ which coincide on $\phi_{0}(E)$ are identical; $\phi_{0}(E)$ generates $G(E) ; G(E)$ is uniquely determined by $E$.

Similar properties hold for $G A(E)$ and $G C(E)$. The uniqueness shows that $G A(E) \approx G(E) / C, C$ being the closure of the commutator subgroup of $G(E)$. If $E$ is discrete, $G(E)$ and $G A(E)$ are the classical free group and free abelian group generated by the set $E$.

THEOREM 1. A necessary and sufficient condition for $\phi_{0}$ to be $a$ homeomorphism is that $E$ be completely regular. The uniform structures induced on the $\phi_{0}(E)$ are the universal uniform structure (for $G(E)$ and $G A(E)$ ), and the Cech uniform structure (for $G C(E)$ ).

The necessity is clear. The sufficiency comes easily from the fact that one has sufficiently many mappings continuous of $E$ into the real line $R$ or the unit circle $T$.

THEOREM 2. If $E^{\prime}$ is a subspace of the completely regular space $E$, $G\left(E^{\prime}\right), G A\left(E^{\prime}\right)$ and $G C\left(E^{\prime}\right)$ are algebraically isomorphic to subgroups of $G(E), G A(E)$ and $G C(E)$, and have finer topologies.

If we extend the injection $i: E^{\prime} \rightarrow E \rightarrow G(E)$, we get a continuous homomorphism $g: G\left(E^{\prime}\right) \rightarrow G(E) . g$ is shown to be one-to-one by proving that, given a word $\prod_{i} a_{i}^{n_{i}}\left(a_{i} \in E^{\prime}\right)$, there exists a topological group $G$ and a continuous mapping $f$ of $E^{\prime}$ into $G$ such that $\prod_{i} f\left(a_{i}\right)^{n_{i}} \neq I$. One may take for $G$ the unimodular orthogonal group $\mathrm{O}_{3}[R]$ which contains free groups with as many generators as desired, and which is arcwise connected. For $G A\left(E^{\prime}\right)$ an $R^{n}$ is good enough.

As corollaries, we have:

(a) $G(E)$ and $G C(E)$ (resp. $G A(E)$ ) have the algebraic structure of 
the free (resp. free abelian) group generated by the underlying set of $E$.

(b) The commutator subgroup $C$ of $G(E)$ is closed; $G A(E) \approx G(E) / C$.

(c) $\phi_{0}(E)$ is a closed subset of $G(E)$ (resp. $G A(E), G C(E)$ ).

((c) is proved by imbedding $E$ in a compact space.)

(d) Every free group is algebraically isomorphic with a subgroup of a compact group.

(e) The topology of $G(E)$ is finer than the topology of $G C(E)$.

When $E$ is connected, the subgroup $A$ of $G(E)$ (resp. $G A(E)$, $G C(E)$ ) composed of all the words of total degree zero is the connected component of the identity. If $E$ is compact, $A$ is a countable union of compact sets.

If $E^{\prime} \subset E, G\left(E^{\prime}\right)$ is topologically isomorphic with a subgroup of $G(E)$ in the following cases (where continuous mappings of $E^{\prime}$ into sufficiently many groups can be extended to $E$ ):

(a) $E^{\prime}$ is a retract of $E$.

(b) $E$ is the completion of $E^{\prime}$ for the universal uniform structure.

(c) $E$ is normal and $E^{\prime}$ is a closed subset of $E$.

A counter-example is the following: $E^{\prime}$ is a discrete noncountable space, and $E$ is its Cech compactification (consider the subgroup composed of the words of total degree zero).

The Markoff's schemes. By a scheme is meant a set $\mathfrak{S}$ of words formed from elements of $E$. We shall consider the continuous mappings $\left\{f_{\gamma}\right\}$ of $E$ into topological groups such that $\prod_{i} f_{\gamma}\left(a_{i}\right)^{n_{i}=1}$ for every word $\prod_{i} a_{i}^{n_{i}} \in \mathfrak{S}$. Such an $f_{\gamma}$ is called a realization of the scheme $\mathbb{S}$.

We apply the general construction to the mappings $f_{\gamma}$ (with the usual restriction on cardinal numbers): we form the partial product $\prod_{\gamma} G_{\gamma}$ of $\prod_{\alpha} G_{\alpha}$, and construct the subgroup $G \subseteq(E)$ generated by the elements $\left(f_{\gamma}(x)\right)(x \in E)$. Let $\phi_{\mathscr{S}}$ be the mapping $x \rightarrow\left(f_{\gamma}(x)\right)$. It is clear that every realization of the scheme $\subseteq$ may be written $f=g \circ \phi_{\mathscr{S}}$, where $g$ is a continuous homomorphism of $G \mathfrak{S}(E)$. On the other hand such a pair $\left(G \mathfrak{S}(E), \phi_{\mathscr{S}}\right)$ is unique. If $H$ denotes the closure of the invariant subgroup of $G(E)$ generated by $\mathfrak{S}, G \subseteq(E)$ is isomorphic with $G(E) / H$ (because of the uniqueness).

ExAmples. (a) $G(E)$ is defined by the empty scheme, $G A(E)$ by the set of commutators.

(b) If $E$ has a structure of topological group, we may take for $\checkmark$ the "multiplication table" of $E$. A pair having the properties of $\left(G \mathfrak{S}(E), \phi_{\mathscr{S}}\right)$ being ( $E$, identity), we deduce from the uniqueness that:

Every topological group $E$ is isomorphic with a factor group of the free topological group generated by the underlying topological space of $E$.

(c) Taking for $E$ the topological sum of two topological groups (with identity elements identified), and for $\mathfrak{S}$ the union of their multi- 
plication tables, one gets the free topological product of the two groups.

The referee has pointed out to me that S. Kakutani (Free topological groups and infinite direct products of topological groups, Proc. Imp. Acad. Tokyo vol. 20 (1944) pp. 595-598) gives substantially the same proof of Markoff's theorem as I do.

Notice also that Nakayama's results ( $A$ note on free topological groups, Proc. Imp. Acad. Tokyo vol. 19 (1943) pp. 471-475) can be obtained by our method: his topology for $G(E)$, deduced from the continuous representations, is that of our $G C(E)$. If $E$ is a uniform space, Nakayama's uniform free topological group is obtained by taking, as $(S-T)$-mappings, the uniformly continuous ones.

Grenoble, France 